\title{
Dogma statt Debatte. Wirtschaftswachstum im parlamentarischen Diskurs
}

\author{
Manuel Rivera $\cdot$ Franziska Zucher
}

Online publiziert: 20. September 2018

(C) Der/die Autor(en) 2018

Zusammenfassung Wirtschaftswachstum ist als Politikziel zwar gesellschaftlicher Konsens, wird aber auch immer wieder infrage gestellt. Argumente gegen dieses Ziel sind durch die Enquete des 17. Deutschen Bundestages nachvollzogen worden. Ein entsprechender Lerneffekt aber lässt sich auf der Ebene öffentlich sichtbaren parlamentarischen Sprachhandelns nicht beobachten, wie unsere Inhaltsanalyse von 120 offiziellen Dokumenten der 18. Legislaturperiode zeigt. Der von den regierenden Parteien geprägte Diskurs ist eigentümlich randständig (ornamental) und frei von Argumentationen (dogmatisch). Es gibt keine Debatte zum Thema. Dies ist nicht hinreichend durch kommunikative Strategien oder Positionen erklärbar, sondern deutet auf den Effekt eines historisch verfestigten Diskurses. Die Frage stellt sich, inwiefern auch andere nicht-tagesaktuelle Grundsatzfragen im Parlament marginalisiert werden. Methodisch ist für die politische Diskursanalyse außerdem generell das Verhältnis von Zweck und Mittel bei der Aussagenbildung von Interesse.

Schlüsselwörter Inhaltsanalyse - Diskurs · Bundestag · Wirtschaftswachstum • Responsivität

\section{Dogma, not Debate. Economic Growth in Parliamentary Discourse}

Abstract Economic growth as a policy objective remains consensual, but not unchallenged. The enquiry committee set up during the German Bundestag's 17th electoral period has reproduced some of the arguments against said objective. Via con-

\footnotetext{
M. Rivera $(\square)$

Institute for Advanced Sustainability Studies (IASS), Potsdam, Deutschland

E-Mail: manuel.rivera@iass-potsdam.de

F. Zucher

Berlin, Deutschland

E-Mail: franziska.zucher@web.de
} 
tent analysis of 120 official documents from the 18th electoral period, however, we show that this apparent learning process has left no traces at all. Growth discourse is predominantly governmental, weirdly marginal (ornamental) and devoid of argumentative reasoning (dogmatic). These results cannot be sufficiently explained by communicative strategies or positions, instead they point to the effect of a historically consolidated discourse. A resulting question is whether other fundamental, yet notcurrent political issues might be marginalized within parliament, in a similar manner. Furthermore, the general ratio between means and objectives is of methodological interest for political discourse analysis.

Keywords Content analysis · Discourse - German Bundestag · Economic growth • Responsiveness

„Der Grund aller Verkehrtheit in Gesinnungen und Meinungen ist: Verwechselung des Zwecks mit dem Mittel“"

(Novalis [1798] 1984, S. 59)

\section{Einleitung: Wachstum als kritisierbares Politikziel und die Frage nach parlamentarischer Responsivität}

In den Siebzigerjahren geriet, im Gefolge der Ölpreiskrise und der Studie des Club of Rome zu den „Limits to Growth“, auch in der bundesrepublikanischen Öffentlichkeit ein wichtiger politischer Grundkonsens ins Wanken: der zur Möglichkeit und Notwendigkeit stetigen Wirtschaftswachstums. Dieser Konsens war zwar erst Ende der Sechzigerjahre von Karl Schiller im sogenannten Stabilitätsgesetz auf den Schultern einer politischen Großen Koalition und einer rezessionsgeängstigten, auf Wachstum setzenden Medienöffentlichkeit (Knauß 2016, S. 65-74) sowie im Kontext einer Aufwertung der rein wirtschaftswissenschaftlichen (Konjunktur-)Politikberatung fixiert worden. ${ }^{1}$ Die malthusianischen Modellrechnungen von Dennis Meadows und Kollegen fielen dennoch auf fruchtbaren medialen Boden, der vor allem durch Umweltverschmutzungsthemen, aber auch durch das Reden von aus den Nähten platzenden, unwirtlichen Städten und ungewissen Zukünften allgemein bereitet worden war. Wirtschaftswissenschaftlern und ihren journalistischen Adepten gelang es freilich binnen weniger Jahre, die Zweifel am Wirtschaftswachstum durch Innovations- und Substitutionsargumente zurückzudrängen. Seither kann man, was die Printmedien und die durch sie veröffentlichte Meinung betrifft, durchaus von einer ,langen Gegenwart des Wachstumsparadigmas“ sprechen, in der Wachstumskritik zwar zur Kenntnis, aber nicht eigentlich ernst genommen wird (Knauß 2016, S. 75-96, 128-131).

\footnotetext{
$1 \mathrm{Zu}$ nennen ist hier vor allem die Gründung des Sachverständigenrates als unabhängiges Wissenschaftlergremium - im Gegensatz zu korporatistischer Meinungsbildung, die u. U. auch verteilungspolitische Fragen stärker diskutiert hätte (Nützenadel 2002).
} 
Trotzdem gibt es eine bundesdeutsche Wachstumsdebatte. Sie hat diesseits der medialen, also in „kleinen“ bzw. „mittleren“, d.h. Versammlungs-Öffentlichkeiten (vgl. Gerhards und Neidhardt 1993, S. 63) mindestens zwei wichtige Wellen erlebt. Die erste, in den Neunzigerjahren durch den Rio-Gipfel mit ermutigt, fand Inspiration in den neu auflebenden Ecological Economics (Daly 1993) und Ausdruck vor allem in zivilgesellschaftlichen Dialogen und Publikationen (BUND und Misereor 1997). Sie verebbte unter dem Druck der übermächtigen Arbeitslosigkeitsprobleme und des auf sie reagierenden, Wachstum durch neoliberale Strukturreformen zu befördern suchenden Diskurses (Wengeler und Ziem 2010); statt der Agenda $21 \mathrm{kam}$ die Agenda 2010. Die zweite Welle folgte der Finanzkrise ab 2008, als die unterdessen weiterentwickelten wissenschaftlichen Überlegungen zur Qualifizierung und ggf. Einschränkung von Wachstum neue Respektabilität gewannen und sich mit Fragen nach Indikatoren eines ,guten Lebens“ jenseits materieller Wohlstandssteigerung verbanden (Pennekamp 2011). Die gesellschaftliche Diskussion hierzu, die umweltbezogene Argumente der Neunzigerjahre reaktivierte und sie mit globalisierungskritischen Motiven verband (BUND und Brot für die Welt 2010; Brand 2014), wurde so unüberhörbar, dass der Deutsche Bundestag sich genötigt fühlte, eine Enquete-Kommission einzusetzen; ,Wohlstand, Wachstum und Lebensqualität““.

Enquete-Kommissionen können verstanden werden als Versuche des politischen Systems, nicht nur wissenschaftliche Zusatzlegitimation einzuholen oder Politik zu „entlasten“, sondern tatsächlich auch solche gesellschaftlichen Problemlagen ,präsumptiv zu reformulieren sowie mögliche Lösungsansätze aufzuzeigen“, die im System sonst noch nicht artikulierbar sind (Hampel 1991, S. 129). Dabei muss Konsens de facto nicht unbedingt hergestellt werden, ist aber meist ein Ziel der fraktions- und fachübergreifenden Deliberation. Im Falle der Wachstums-Enquete wurde dieses Ziel in vielen Bereichen verfehlt. Es ist aber gleichwohl interessant, danach zu fragen, ob der Kommissionsbericht im Bundestag „Spuren hinterlassen“ hat. Denn auch „das regelgeleitete Prozessieren von Dissens“ kann ,integrative [...] Wirkung entfalten“ (Hampel 1991, S. 130); im vorliegenden Falle könnten also die Frontlinien bezüglich der Machbarkeit von „Entkopplung“, der Wünschbarkeit weiterer Zunahme von materieller Güterproduktion etc., die sich im Enquete-Bericht von 2013 finden, als neue bzw. nun klarer artikulierbare Positionsoptionen in der parlamentarischen Willensbildung fortan eine Rolle spielen. Geklärt werden könnte, mit anderen Worten, worüber man sich politisch weiter auseinanderzusetzen hat: die Themen künftiger Debatten. Auch wenn die Wachstums-Enquete an der Definition solcher ,Verständigungsaufgaben" möglicherweise gescheitert ist (Ueberhorst 2014), so hat sie doch in ihrem Bericht immerhin den Minimal-Konsens etabliert, dass Wirtschaftswachstum „kein Ziel an sich“, sondern nur „Mittel zur Erreichung anderer Ziele“ sein könne (Deutscher Bundestag 2013, S. 24, 589) - eine Hierarchisierung, die man aus der Formulierung des sogenannten Magischen Vierecks im vorerwähnten Stabilitätsgesetz von 1967 zwar herauslesen kann, aber keinesfalls muss. ${ }^{2}$ Damit könnte sie dem

\footnotetext{
2 Das Gesetz hat in erster Linie das ,gesamtwirtschaftliche Gleichgewicht“ im Auge und will ,zur Stabilität des Preisniveaus, zu einem hohen Beschäftigungsstand und außenwirtschaftlichem Gleichgewicht bei stetigem und angemessenem Wirtschaftswachstum beitragen“ (Deutscher Bundestag 1967, § 1, unsere Hervorhebung.).
} 
Diskurs zwar kein Thema, aber eine Art grammatische Regel empfohlen haben: Wachstum zu anderen Politikzielen in Beziehung setzen und seine Angemessenheit zur Erreichung dieser Ziele diskutieren. Den Argumenten pro oder contra Wachstum würde damit eine bestimmte Struktur vorgegeben.

Unsere Studie eruiert, ob diese Thematisierungsstruktur den parlamentarischen Diskurs während der auf die Enquete folgenden Legislaturperiode tatsächlich geprägt hat, und inwiefern dies ein Aufgreifen der o.g. gesellschaftlichen Debatte ermöglichte. Diese Rekonstruktion folgt neben einem thematischen Interesse auch dem politikwissenschaftlichen, ob (und wie) Grundsatzfragen, die nicht primärer Gegenstand der Programmbildung durch Parteien, durchaus aber der gesellschaftlichen Diskussion sind, im Parlament thematisiert werden. Dies ist zunächst eine Frage nach der sogenannten Repräsentations- und Kommunikationsfunktion des Bundestages. Zu ihr zählt nicht nur das Herstellen bzw. Führen von Öffentlichkeit, sondern zuvörderst auch die Responsivität, d.h. Inputaufnahme und -verarbeitung. Unsere triangulierte Untersuchung, die Tiefeninterviews mit Abgeordneten, eine standardisierte Befragung ihrer Mitarbeiterinnen ${ }^{3}$ und eine Inhaltsanalyse von Bundestagsdokumenten einschloss, erlaubt es uns, hier etwas zu beobachten, das in der Parlamentarierforschung akteurstheoretisch als Riss in der „Responsivitätskette“ bezeichnet worden ist: Beim Zusammenführen der Inputs der fachlich spezialisierten „einfachen Abgeordneten“ verhindern unter anderem starke Hierarchien in den Fraktionen eine adäquate Meinungs-Aggregation (von Oertzen 2006, S. 283-285). Wir haben zu entsprechenden Mechanismen, das Wachstumsthema betreffend, durch unsere MdB-Interviews ebenfalls Hinweise erhalten - neben jenen der Fraktionshierarchien beispielsweise den altbekannten Topos der alltäglichen politischen Zeitnot (Palfner 2006, S. 226; Bohrmann und Laux 2018) oder den Umstand, dass wachstumskritische Motive zu wenig von Gewerkschaften mitgetragen werde, die stärker als jede andere Gruppe das Ohr des Bundestags haben (Vogel 2014). Doch wie sehr man solche Erklärungsansätze auch aufsummiert: Sie scheinen zu schwach für den empirischen Befund der fast völligen Verneinung unserer Ausgangsfrage. Wachstum wird im Bundestag sehr selten als Mittel für Politiken kommuniziert, sondern fast immer als Zweck, und argumentiert wird für oder gegen diesen Zweck kaum jemals. Der dogmatische Eindruck, den die entsprechende „Gebetsmühle“ erweckt, führt dazu, die Frage nach dem Diskurs hinter den Kommunikationen methodologisch neu zu formulieren: In den Verfestigungen des Textmaterials erscheint eine Art „Exteriorität" des Diskurses gegenüber seinen in Bezug auf das Thema durchaus plural orientierten Sprecherinnen, die durch eher struktural operierende, Foucaultsche Ansätze der Diskursanalyse als durch einen neuerlichen Bezug auf die Akteure erklärt sein will (Kerchner 2006, S. 56-57). Dabei kann die grammatische Unterscheidung zwischen Zweck und Mittel solche Ansätze unseres Erachtens gerade im politischen Bereich systematisch bereichern.

Im Folgenden erläutern wir die methodische Anlage unserer Studie „Growth in Politics“, insbesondere ihres inhaltsanalytischen Teils; dabei gehen wir auch auf den zunächst von uns verwendeten Diskursbegriff und seine eingeschränkte Brauch-

\footnotetext{
${ }^{3}$ Im Sinne einer gendergerechten Sprache verwenden wir in diesem Artikel weibliche und männliche Formen unregelmäßig alternierend.
} 
barkeit ein, also auf die Grenzen, an die unsere Methodik stieß (Abschn. 2). Diese Grenzen explizieren wir anhand unserer Ergebnisse, insofern diese den Wachstumsdiskurs im Bundestag als randständig, gewissermaßen „ornamental“ ausweisen (Abschn. 3), und spüren der spezifischen Reproduktionsweise des Dogmas „Wachstum als Politikziel“" durch die Zweck-Mittel-Grammatik nach (Abschn. 4). Dabei lassen sich natürlich auch Differenzierungen beobachten, etwa in einigen Versuchen vor allem seitens der Opposition, Wachstum als Ziel einzuschränken oder zu kontextualisieren, aber auch in gewissen konjunkturellen Schwankungen seiner Prominenz (Abschn. 5). ${ }^{4}$ Abschließend bilanzieren wir die Aussichten der politischen Wachstumsdebatte und skizzieren eine methodische Anregung an die politische Diskursforschung (Abschn. 6).

\section{Der parlamentarische Wachstumsdiskurs: Begriff, Datenbasis und Methodik}

Der Thematisierung von Wachstum im Deutschen Bundestag näherten wir uns 2015/2016 explorativ, mit Hilfe eines Mixed-Method-Designs. Neben Bundestagsdokumenten untersuchten wir die Wachstums- und Wohlstandsauffassungen sowie das Informationsverhalten einzelner Mitglieder des Bundestags (MdBs) auf Basis von Leitfadeninterviews; zudem befragten wir die Mitarbeiter der MdBs mit Hilfe eines standardisierten Fragebogens über den Meinungsbildungsprozess im parlamentarischen Alltag sowie ihre individuellen Einstellungen zu Wirtschaftswachstum. Dabei interessierte uns unter anderem, in welchen fachpolitischen Bereichen eine Wachstumsdebatte überhaupt anschlussfähig ist, welche wachstumskritischen Argumente dabei verarbeitet (also ggf. auch zurückgewiesen) werden und welchen Einfluss auf entsprechende Einstellungen der MdBs die üblichen Prädiktoren wie Parteizugehörigkeit oder Seniorität, aber möglicherweise auch informelle Faktoren wie eine bestimmte Medienrezeption oder die Meinungsbildung im Büroalltag gewinnen. Der Dokumentenanalyse war dabei in der Triangulation ursprünglich die Funktion zugedacht, das öffentliche Sprachhandeln von Parlamentarierinnen objektiv zu beobachten, um den in ihm sich realisierenden Sprecherintentionen per Interview auf den Grund zu gehen und um umgekehrt die Antworten der Büromitarbeiter auf standardisierte Fragen im Lichte der in den Dokumenten explizierten Argumente genauer interpretieren zu können.

\footnotetext{
4 Bereits die Jenaer Parlamentarierstudie hatte, als sie die Abgeordneten des 15., 16. und 17. Deutschen Bundestages nach einer Priorisierung von Wachstumsförderung vs. Ungleichheitsbekämpfung vs. Fiskalstabilität fragte, mehrfache Verschiebungen von Gewichtungen festgestellt (Best et al. 2010, S. 12); wie bei uns auch war dabei ein Parteieinfluss zwar überwältigend prädiktiv, es fanden sich aber auch geschlechtsund regionalspezifische Unterschiede (Best und Vogel 2012, S. 42-43).
} 
Dass wir im vorliegenden Beitrag auf Erkenntnisse aus den Befragungen nur gelegentlich eingehen, ist nicht nur pragmatischer Fokussierung geschuldet. ${ }^{5}$ Es hat seinen Grund vor allem auch darin, dass die geplante Verwendung der Bundestagsdokumente als „Deutungshorizont“ in der Studie empirisch scheiterte - und mit ihr, so lässt sich sagen, unser theoretisch zumindest implizit zugrunde gelegter ,,interaktionistischer" Diskursbegriff. Wir hatten uns zunächst auf eine akteurszentrierte, dem Alltagsgebrauch des Begriffes angenäherte Definition von Jürgen Gerhards gestützt. Ihr zufolge bezeichnet der Diskurs „öffentlich geführte Kommunikationen von Akteuren über Themen, darauf bezogene Positionen, Begründungen und Deutungen“ (Gerhards 2010, S. 334). Diese Kommunikationen finden in Foren statt; auch das Parlament kann als ein solches bezeichnet werden. Hier agieren individuelle oder kollektive Akteure (in unserem Falle Fraktionen, Ausschüsse, die Bundesregierung und einzelne Abgeordnete) mit ihren kommunikativen Beiträgen in einer "Arena“. Sie sind ausgerichtet auf eine „Galerie“, die Vorgänge in der Arena beobachtet und bewertet; auf der parlamentarischen Galerie bewegen sich parlamentarische Akteure selbst (z. B. die Fraktionen gegenüber den Berichten der Ausschüsse), aber natürlich auch außerparlamentarische wie Verbände, NGOs, einzelne Bürger sowie die Medien. Darüber hinaus verfügt jede Arena über eine Backstage: die Ressourcen und die Beziehungsmuster zwischen den Akteuren (Gerhards 2010, S. 334); hierüber wollten wir über Fragen an MdBs und ihre Mitarbeiter nach Kommunikationsroutinen im Büroalltag Aufschluss gewinnen.

Ein solches interaktionistisches, Kommunikationen in abgrenzbaren Öffentlichkeiten beschreibendes und teils aus anderen Kommunikationen, teils aus Dispositionen der Sozialstruktur erklärendes Diskursverständnis ist nicht nur gut kombinierbar mit primär forschungspragmatischen Überlegungen zur themenorientierten, zeitraumbezogenen Eingrenzung von Diskursen als virtuellen Aussagekorpora mit intertextuellen Verweisungen (Busse und Teubert 1994, S. 14). Als ,,analytischpragmatisches“, vor dem Hintergrund der in Abschn. 1 benannten Frage nach parlamentarischer Responsivität teilweise auch ,normativ-kritisches“ Herangehen an Diskurse mit einem besonderen Interesse an Argumentationen (Kerchner 2006, S. 38, 50-51) ließ es auch erwarten, Zusammenhänge zwischen Strategien und Akteuren beobachten zu können. So wäre z. B. vorstellbar gewesen, dass in dem die Auseinandersetzung ermöglichenden ,Diskursfeld“ Bundestag bestimmte das Wirtschaftswachstum auf ,,saubere“ Produktionsformen einschränkende und es damit als „Green Growth" konzipierende Aussagen durch MdBs des Wirtschafts- oder aber des Umweltausschusses stärker vertreten und von ihnen ,diskursgemeinschaftlich“ in der Auseinandersetzung mit bestimmten Lobbygruppen oder Medien, ggf. auch dem vorerwähnten Enquête-Bericht reproduziert und in die parlamentarische Willensbildung agonal eingebracht werden (Schwab-Trapp 2011, S. 291-293). Das kursiv Gesetzte war aber nicht der Fall. Zwar fanden wir in den Befragungen qua Korrela-

\footnotetext{
5 Einen Gesamtabriss der Studie haben wir in einem Working Paper gegeben (Rivera et al. 2016). Ein kürzlich erschienener Artikel in der Zeitschrift Futures referiert einige der inhaltsanalytischen Resultate, legt den Schwerpunkt jedoch auf eine historische Einordnung derselben sowie ihr Verhältnis zur DegrowthKommunikation (Rivera 2018). Intensiv auf den modus operandi der Bundestagsdokumente in den verschiedenen parlamentarischen Teilarenen konzentrieren wir uns lediglich im vorliegenden Artikel.
} 
tionen von Einstellungsitems und unabhängigen Variablen solche Zusammenhänge auf, zusammen mit einer hohen Meinungspluralität und einem noch höheren Wunsch nach pluralen Debatten, ${ }^{6}$ in den offiziellen Bundestagsdokumenten aber kamen wir mit diesem Beobachtungsansatz nicht weiter. Dort stießen wir vielmehr auf eine „,institutionell verfestigte Redeweise“ (Link 1983, S. 60), die das öffentliche parlamentarische Sprachhandeln bestimmt und offenbar zumindest insofern eine von Sprecherintentionen entkoppelte spezifische Macht ausübt, als sie die Artikulation von Argumentationen zu Wachstum überhaupt unwahrscheinlich macht.

Dass und wie solches strukturelle Marginalisieren von Argumenten geschieht, will dieser Artikel besonders im dritten und vierten Abschnitt plausibel machen. Dabei geraten nicht Strategien einzelner Akteure oder Diskursgemeinschaften in den Blick, sondern vor allem ihre ,Zerstreuung [...] und Diskontinuität“" in einem „Raum der Exteriorität“ (Foucault 1972, S. 55), der über konfligierende Geltungsansprüche hinausreicht. Das „Verteilungssystem“ von Wachstumsaffirmationen, das an ihre Stelle rückt und dabei andere Aussagen ausschließt, und das man als diskursive Formation bezeichnen kann (Foucault 1972, S. 28-29, 38), ist in unserem Falle das Wachstumsparadigma, d.h. die Auffassung von einem durch das Bruttoinlandsprodukt (BIP) angemessen abgebildeten, fortwährenden Wirtschaftswachstum als Kernbestandteil sozialen Fortschritts und sozialer Wohlfahrt (Schmelzer 2015, S. 264). Es liegt nahe, die Herausbildung und - im Falle der vorliegenden Studie - die Reproduktion ${ }^{7}$ dieser Formation mit Laclau und anderen als ,hegemonial“ zu beschreiben - wobei wir feststellen werden, dass es für eine befriedigende Beschreibung des parlamentarischen Wachstumsdiskurses in diesen Termini eines entscheidenden Definiens ermangelt, nämlich des gemeinsam vertretenen Antagonismus gegenüber einem Außen, der „Kontrarität“ (Laclau und Mouffe 1991, S. 161-167; Nonhoff 2010, S. 314).

Forschungstechnisch haben wir uns im Sinne des eingangs beschriebenen Interesses auf die qualitative Inhaltsanalyse im Sinne eines teils konzeptgeleiteten, teils induktiven Vorgehens gestützt (Früh 2007, S. 154), das eine partielle Quantifizierung der Befunde für zentrale Kategorien zumindest ermöglicht (Mayring 2010, S. 21). Darüber hinaus waren wir auf den Spuren des von der Enquete etablierten Konsenses interessiert an einer „Grammatik“ im Sinne von Kenneth Burke: geleitet also von der Frage nach einem ganz bestimmten grammatischen „Verhältnis“ (ratio), nämlich dem von Zweck (purpose) und Mittel (agency) (Burke 1968, 1978). Rückblickend halten wir diese in unserm Falle vor allem empirisch begründete Perspektive für eine methodische Neuerung, die gerade für eine eher poststrukturalistisch orientierte Diskursanalyse interessant sein könnte.

\footnotetext{
${ }^{6}$ Als direktester Ausdruck dieses Wunsches lässt sich lesen, dass $83 \%$ der MdB-Mitarbeiter die Notwendigkeit einer „Debatte über alternative Wachstumskonzepte“ bejahten, teilweise mit inhaltlichen, aber eben auch mit prozeduralen, demokratiebezogenen Argumenten wie dem, dass nur Debatten „Alternativen aufzeigen oder gar lebendig werden lassen“".

7 Zur umfassenden Untersuchung beider Dynamiken hätte unsere Analyse freilich eines diachroneren Korpus bedurft, den man für eine instruktive Diskursanalyse als Prozessanalyse als unabdingbar betrachten mag (Schwab-Trapp 2010, S. 178).
} 
Unsere Analyse des parlamentarischen Diskurses hinsichtlich wachstumsbezogener Argumente erfolgte anhand einer Auswahl von Bundestagsdokumenten, die in der 18. Legislaturperiode zwischen Oktober 2014 und Ende Januar 2016 veröffentlicht wurden. Die Auswahl war von den weiter oben in diesem Abschnitt angedeuteten Vermutungen über Kommunikationszusammenhänge geleitet und orientierte sich an (1) den Funktionen des Deutschen Bundestages, (2) der Verwendung des Wachstumsbegriffs, (3) der Kohärenz parlamentarischer Vorgänge sowie (4) den Kriterien Fraktion und Fachpolitik. Um so z.B. ad (1) Gesetzgebung, Kontrolle und Repräsentation gleichermaßen in den Blick zu bekommen, berücksichtigten wir Große und Kleine Anfragen, Anträge der Fraktionen, Berichte und Beschlussempfehlungen der Ausschüsse, Unterrichtungen durch die Bundesregierung, Regierungserklärungen und Plenarprotokolle. Dabei bevorzugten wir Dokumententypen, in denen die Möglichkeit der Argumentation gegeben ist, bevorzugten daher z.B. Beschlussempfehlungen der Ausschüsse gegenüber Verordnungen. Zudem wählten wir ad (2) innerhalb dieser Dokumentengruppen vorwiegend solche Dokumente aus, die den Begriff „Wachstum“ enthalten, und steuerten nur insofern bei einigen Gruppen, als wir zusätzlich Dokumente aufnahmen, in denen der Wachstumsbegriff selbst nicht auftaucht, wirtschaftliche Themen jedoch sehr präsent waren. Dies taten wir, um sicherzustellen, dass keine wichtigen wachstumsbezogenen Argumentationsmuster übersehen werden - eine im Nachhinein sich als weitgehend unnötig herausstellende Vorkehrung.

Ähnlich steht es um den Nachvollzug parlamentarischer Interaktionen, um dessentwillen wir ad (3) den Anfragen die dazugehörigen Antworten der Bundesregierung sowie den ausgewählten Regierungserklärungen die ihnen folgenden Plenardebatten zur Seite stellten. Auch er erwies sich - mit sehr wenigen, weiter unten sämtlich erwähnten Ausnahmen - als analytisch kaum ergiebig. Schließlich wollten wir ad (4) möglichen Einflüssen von Policy und Fraktionslinie auf Problemverständnisse Rechnung tragen; besonders letztere ist ein starker Prädiktor in der akteursorientierten Bundestagsforschung (Heinemann und Janeba 2010). Daher achteten wir bei der Auswahl insbesondere von Berichten und Beschlussempfehlungen sowie von Anträgen auf ein ausgewogenes Spektrum der Ausschüsse bzw. Fraktionen. So sollte die Auswahl der Dokumente zumindest eine Analyse der Unterschiede (und Gemeinsamkeiten) zwischen Opposition und Regierung bzw. zwischen Linken und Grünen ermöglichen, und in der Tat fand sich hier ein gewisser, wenn auch im Gesamteindruck der Daten nicht gerade überwältigender Effekt. All dies ist insbesondere Thema in Abschn. 5.

Insgesamt codierten wir mithilfe der Analysesoftware MAXQDA 120 Texte mit 1825 Markierungen (Codings). Dabei griffen wir zwar hin und wieder auch auf quantitativ-lexikalische Elemente insbesondere zur Groborientierung zurück, sahen uns jedoch insgesamt, wie oben bereits ausgeführt, einem interpretativen Ansatz verpflichtet und unterscheiden uns somit stark von Untersuchungsdesigns, die sich an Text Mining orientieren und/oder diktionärsbasiert arbeiten (wie in dieser Zeitschrift Blätte und Wüst 2017). Solche Ansätze mögen zwar einer poststrukturalistischen Diskursanalyse prima facie näher stehen (Jung 2011, S. 50), jedoch war dieser Ansatz ja gar nicht in unserem Sinne. An sprecherbasierten Argumentationen 
interessiert, ${ }^{8}$ strebten wir im Codierprozess eine Aggregation auf wenige Obercodes an, um die entscheidenden argumentativen Linien auf einen Blick erfassen zu können, so zum Beispiel die bereits erwähnte Unterscheidung zwischen Wachstum als Mittel und Wachstum als Zweck. Dass wir durch das letztgenannte, dem EnqueteBericht entnommene und deduktiv auf das Korpus angewandte Strukturelement auf eine „Aussage“ (Palfner 2006, S. 213-216) stoßen würden, die gerade keine Differenzierung zwischen Sprechern zuließ, ist Thema vor allem in Abschn. 4. Zunächst aber wenden wir uns einem anderen Aspekt zu, der Ornamentalität des Diskurses.

\section{Ornamentalität des Diskurses}

Unser Korpus stellte einen für unsere Forschungsfrage relevanten Querschnitt durch den parlamentarischen Diskurs der 18. Legislaturperiode dar. Obwohl wir dabei durchaus an der Wachstumsthematisierung orientiert waren, handelt es sich bei diesem Querschnitt gleichwohl nur bedingt um einen Wachstumsdiskurs im Sinne ,thematisch verknüpfter Aussagenkorpora“" (Jung 2011, S. 47, eigene Hervorhebung). Denn obwohl in den meisten der Dokumente Wirtschaftswachstum erwähnt wird und in den anderen eine Erwähnung wegen wirtschaftspolitischer Themen erwartbar gewesen wäre, sind diese Dokumente doch keinesfalls um die Thematik Wirtschaftswachstum herum zentriert oder gar dadurch verbunden. Dass eine entsprechende Auswahl für den Bundestag gar nicht möglich gewesen wäre, ist selbst ein empirischer Befund. Bereits zu Beginn des Auswahlprozesses fiel auf, dass Wachstum als Thema, akteursbezogen ausgedrückt, den Parlamentariern nicht gerade unter den Nägeln brennt. Der Begriff taucht im Untersuchungszeitraum insgesamt nur in knapp acht Prozent aller Dokumente auf. ${ }^{9}$ Dabei gibt es ein vielfaches relatives Übergewicht des Vorkommens in den Plenardebatten sowie in den in einige dieser Plenardebatten integrierten, aber auch einzeln analysierbaren Regierungserklärungen (je $73 \%$ ), gefolgt von einer deutlichen Ausprägung in den Unterrichtungen der Bundesregierung (29\%). Es sind also vor allem von der Regierung als zentraler Sprecherin geprägte Arenen, in denen Wachstum vorherrscht. Die Unterrichtungen gehen zwar auf Berichtspflichten und somit auch auf die Kontrollfunktion des Parlaments zurück, werden aber ausschließlich durch die Ressorts kontrolliert und erzeugt. Un-

\footnotetext{
${ }^{8}$ In diesem Sinne ähnlich nicht nur wie die weiter oben zitierten Autoren Gerhards und Schwab-Trapp, sondern auch wie Maarten A. Hajer, der in seiner klassischen Untersuchung zum Diskurs über den Sauren Regen die verschiedenen Storylines der Institutionen als argumentativ gesicherte Ereignisfolgen rekonstruierte, mit denen Akteure in ihrem Kampf um Hegemonie ,,versuchen, sich Unterstützung für ihre Definition der Wirklichkeit zu sichern“ (Hajer 1995, S. 59). Als Abkürzungen oder Signa solcher Storylines kann man „Frames“ beschreiben (Lakoff 2008), auf die wir in Abschn. 5 eingehen.

9 Stichtag war der 28.01.2016. Mit Wachstum ist natürlich nicht in allen Dokumenten Wirtschaftswachstum gemeint, so z. B. wenn vom „Wachstum sozialversicherungspflichtiger Arbeitsverhältnisse“ die Rede ist (ein eigentlich schiefer Ausdruck für „Zunahme“, vgl. BT 18/7324) oder, noch evidenter, wenn in Bezug auf Feinpartikel davon gesprochen wird, sie gefährdeten bei Jugendlichen „,das Wachstum der Lunge“ (18/7394). Unserer Schätzung nach machen solche Verwendungen von Wachstum abseits der Denotation „Wirtschaftswachstum“ jedoch deutlich unter zehn Prozent aller gefundenen Dokumente aus. Die Auszählungen sind nachvollziehbar unter pdok.bundestag.de bzw., die Regierungserklärungen betreffend, unter www.bundesregierung.de; unsere letzten Abrufe stammen vom 21.12.2016.
} 
Abb. 1 Verteilung der Wachstums-Codings auf die Dokumentengruppen (Anzahl Codings je 10.000 Wörter)

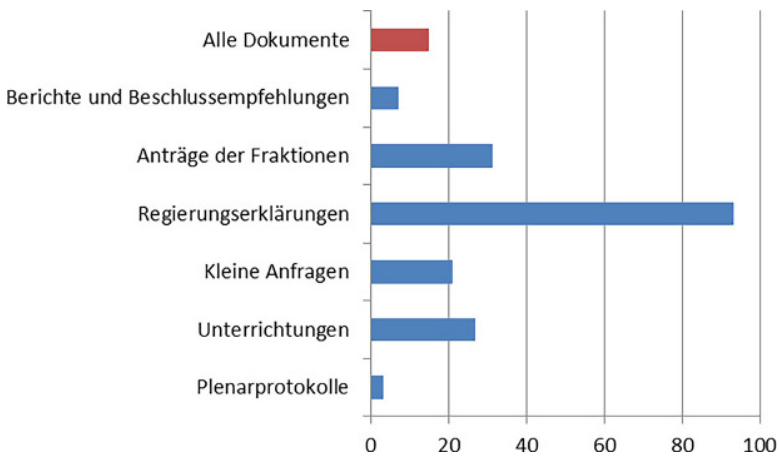

terdurchschnittlich ist das Vorkommen des Begriffs hingegen in Kleinen Anfragen (Regierungskontrolle) und Berichten und Beschlussempfehlungen der Ausschüsse (Gesetzgebung). Das Reden von Wachstum erscheint so zunächst vor allem als Teil der Öffentlichkeitsfunktion, des Darstellens nach außen.

Wenn wir in das von uns thematisch feiner selektierte Textkorpus schauen, und zwar auf alle wachstumsbezogenen Sinneinheiten (Codings) ${ }^{10}$, gewinnen wir ein Bild von der Dichte der Thematisierung in denjenigen Dokumenten, in denen Wachstum erwähnt wird (vgl. Abb. 1). Dabei bestätigt sich, dass Wachstum insbesondere im Herz der Gesetzgebung, also in den Ausschussempfehlungen, kaum eine Rolle spielt, und dass es auch in den die Regierung über den Einbezug von fachöffentlichen Galerien kontrollierenden Kleinen Anfragen (Siefken 2010) nicht unbedingt übermäßig präsent ist. Bereits an den Worthäufigkeiten gerade der Berichte und Beschlussempfehlungen der Ausschüsse etwa sieht man, dass sehr konkrete Aspekte der gegenwärtigen Wirtschafts- und Energiepolitik gegenüber der Reflexion auf Konzepte und Werte dominieren (,Strom“ wird 1201 Mal genannt, das EEG 607 Mal, Wachstum hingegen nur 21, Innovation 16 Mal). In den Worten eines von uns interviewten Haushälters:

Es ist jetzt nicht so, dass irgendwie argumentiert wird: Wir müssen jetzt Gesetz $X$ machen um das Wachstum zu verbessern [...] Es sind ein paar Elemente aus dem Phrasenschwein vielleicht, für Plenarreden oder so.

Allerdings fällt auch in den Plenardebatten ein, aufs Ganze gesehen, sehr spärlicher Gebrauch von Wachstumsargumenten ins Auge, obwohl hier das Wort Wachstum, sucht man erneut nur nach dem unmittelbar wirtschafts- und energiepolitischen Vokabular, eine gewisse Prominenz besitzt (mit 203 Nennungen direkt hinter dem Spitzenreiter „Unternehmen“ mit 224 und deutlich etwa vor „Strom“ mit nur 48 Nennungen). Die Vermutung liegt nahe, dass sich die Wachstumsbezüge auf offizielle Deklarationen beschränken; diese Vermutung bestätigt sich in der Analyse einzelner

\footnotetext{
10 Diese Sinneinheiten sind nicht identisch mit Passagen bzw. einzelnen Nennungen. Eine Passage kann vielmehr verschiedene Aussagen auf einmal treffen bzw. verschiedene relevante Bezüge herstellen. Überdies sind auch implizite Bezugnahmen erfasst. Es gibt also deutlich mehr Codings als explizite WachstumsNennungen.
} 
Debattenverläufe (siehe Abschn. 5). Wachstumsargumente werden weder dafür verwendet, das Pro und Contra von Gesetzgebungsakten zu begründen, noch wirklich dazu, sich im auf die Medienöffentlichkeit ausgerichteten Streit zwischen Regierung und Opposition bezüglich konkreter Politiken zu positionieren. Der Diskurs erscheint also erstens eigentümlich fragmentiert und zweitens kaum funktional; man könnte für beides zusammenfassend auch sagen: Er trägt ornamentalen Charakter.

Diese Ornamentalität ist ein Befund, den es zu interpretieren gilt. Offenbar wird Wachstum, das einst eine bewusst gewählte, emphatisch bejahte strategische Option zur Reduzierung von Verteilungskämpfen war (vgl. Knauß 2016, S. 42-51), heutzutage im Bundestag eher am Rande der Debatten ,mitgeschleift“. Auf welche Weise und mit welcher Pointe dies geschieht, betrachten wir im nächsten Abschnitt.

\section{Die dogmatische Formation: Wachstum als Selbstzweck und Entwicklungssynonym}

Der kleinste gemeinsame Nenner der Enquete-Kommission der Bundesregierung, dass Wachstum kein Ziel an sich, sondern Mittel zur Erreichung anderer Ziele sein solle (siehe oben, Abschn. 1), ist in der diskursiven Alltagspraxis des Parlaments noch lange nicht angekommen. Wenn Wachstum thematisiert wird, geschieht dies kaum als Mittel zur Erreichung anderer Ziele, sondern mit einer überwältigenden Dominanz im Sinne eines politischen Zieles an sich. Die Bilanz lautet 1009 Codings (Zweck) zu 86 (Mittel); ca. 92\% der entsprechenden Sinnzuschreibungen beziehen sich also auf Wachstum als Selbstzweck.

Dieser Befund gilt für die Gesamtheit der untersuchten Texte. Unterschiede zwischen den Dokumententypen gestalten sich so, dass die Häufigkeit der Wachstumals-Selbstzweck-Konstruktionen dieselbe Rangfolge ergibt wie die der Thematisierungsdichte insgesamt (vgl. Abb. 1); die Affirmation von Wachstum als Ziel ist also dort am stärksten ausgeprägt, wo von Wachstum am meisten die Rede ist. Die Analyse der Anträge der Fraktionen offenbart, dass besonders häufig die Regierungsfraktionen Wachstum als Zweck von Politik darstellen. Der Diskurs ist also „gouvernemental“ - nicht in dem von Foucault geprägten, in der Politikwissenschaft gebräuchlichen Sinn eines bestimmten selbstauferlegten Stils des Regierens (vgl. Bröckling et al. 2000), sondern im wörtlichen Sinn eines von der Regierung angewandten Stils.

Die gouvernementale Rhetorik unterliegt dabei in ihrer Intensität auch Schwankungen, wie im Untersuchungszeitraum am Kontrast zwischen den Regierungserklärungen des Wirtschaftsministers zum Jahreswirtschaftsbericht zu beobachten ist. 2014 war ein Jahr höheren Wachstums nach einer „Durststrecke“ (mit Raten unter einem Prozent) gewesen; gleichwohl lag dieses Wachstum unter den vorherigen Prognosen des Sachverständigenrates. Entsprechend offensiv strich der Minister unter diesen Bedingungen den Erfolg Deutschlands auch im Verhältnis zu anderen Ländern heraus; der Wachstumsbegriff findet sich eingebettet in eine europapolitisch aufgeladene, recht pauschale Standortsemantik. Ein Jahr später - die Flüchtlingskrise war in aller Munde, das Wachstum jedoch viel höher als erwartet ausgefallen findet sich der Begriff nur noch am Rande; stattdessen dominieren Referenzen auf 
die deutsche Realwirtschaft und Politik wie „Investitionstätigkeit“, „Mittelstand“ oder das Adjektiv ,,sozial“. Liegt hier das Wachstum als Bezugspunkt in der Sache durchaus nahe, so ist es stilbildend auch in ganz anderen Dokumenten insofern, als der Begriff immer wieder als normative Referenz andere Argumentationen flankiert und so in Gestalt einer Hochwert-Floskel die verschiedensten Themen durchzieht. Dies betrifft zahlreiche Debatten, die sich am tagespolitschen Geschehen orientieren (z.B. Griechenland, TTIP, Europapolitik).

Drei sprachliche Muster, die im Folgenden näher erläutert werden, tragen dazu bei, dass Wachstum im parlamentarischen Handeln nicht nur immer wieder - indirekt - als Zweck von Politik affirmiert, sondern auch Kritik daran unwahrscheinlich wird. Das folgende Zitat ist exemplarisch sowohl für die Indirektheit der Affirmation als auch für das häufigste sprachliche Muster: die Behauptung von Faktoren, die zu Wachstum beitragen bzw. dafür notwendig sind:

Dabei dürfen notwendige Investitionen, Strukturreformen und Haushaltskonsolidierung nicht als Gegensätze, sondern als notwendige Elemente einer wachstumsfördernden Politik verstanden werden. $(18 / 4426)^{11}$

Ein solches Muster, das strittige politische Maßnahmen oder gesellschaftliche Faktoren dem Oberziel Wachstum gewissermaßen en passant unterordnet, prägt in unserem Korpus deutlich über die Hälfte der entsprechenden Codings. Am häufigsten werden dabei Investitionen genannt, gefolgt von Binnennachfrage, Innovationen, Strukturreformen und einem ausgeglichenen Haushalt.

Die zweithäufigste Art und Weise, Wachstum als Zweck von Politik zu etablieren, ist, Wachstum in einem Atemzug mit anderen Politikzielen zu nennen, z. B. in der überaus populären Formel „Wachstum und Beschäftigung“, bei der meist unausgesprochen bleibt, wie das eine mit dem anderen zusammenhängt. Den Arbeitsplätzen als meistgenanntem gleichrangigen Ziel folgen in erheblichem Abstand Investitionen, Wettbewerbsfähigkeit und Wohlstand. Solch eine Verwendung des Wachstumsbegriffs als unhinterfragtes Ziel von Politik macht deutlich, wie Wachstum als politischer Leitbegriff genutzt wird und den ,,guten Ton“ in der (Regierungs-)Politik quasi mitdefiniert. Das Paradigma, das im Wirtschaftswachstum ein Allheilmittel für gesellschaftliche Probleme und einen Maßstab für Fortschritt und Wohlstand sieht, gehört als rhetorisches Element insbesondere bei wirtschaftspolitischen Themen einfach mit dazu. Dies wird auch dadurch deutlich, dass der Bezug zum Wirtschaftswachstum häufig am Beginn der Dokumente, sozusagen als Einstieg in das Thema, erfolgt und im weiteren Verlauf nicht mehr hergestellt wird.

Beide sprachlichen Muster, das Hinzuziehen von Mitteln für Wachstum und die Nebeneinanderstellung von Wachstum mit anderen Zielen, werden sehr oft auch gleichzeitig angewandt, und erneut besonders häufig im Fall des gleichrangigen Ziels „Arbeitsplätze“. Als zwei Beispiele hierfür mögen die folgenden Auszüge aus dem Themenbereich TTIP stehen, die wir jeweils dem Jahreswirtschaftsbericht 2015 und einer Kleinen Anfrage der Fraktion von Bündnis 90/Die Grünen entnommen

\footnotetext{
11 Zitiert werden die Bundestagsdokumente gemäß ihrer Drucksachen-Nummer; sie sind über www. bundestag.de jederzeit abrufbar.
} 
haben und in denen Wettbewerb bzw. offene Märkte als das Mittel der Wahl für mehr Wachstum fungieren:

Ziel ist es, die Märkte auf beiden Seiten des Atlantiks stärker zu öffnen und damit zu Wachstum und mehr Beschäftigung beizutragen. (18/3840)

Eines der Hauptargumente für den Abschluss eines ambitionierten Abkommens ist das Versprechen, dadurch Wirtschaftswachstum zu generieren und Arbeitsplätze zu schaffen. (18/0828)

Es ließe sich natürlich mutmaßen, dass in solchen Fällen ,gemeint“" sei, dass Wachstum zu Arbeitsplätzen beiträgt und dass diese daher als ,eigentliches“ Oberziel der Politik stehen. Indes würden solche intentionsbezogenen Spekulationen den methodischen Punkt einer objektivierenden Inhaltsanalyse verfehlen. Interessant ist vielmehr gerade, dass in der Grammatik des Diskurses Wachstum als selbstständiges, den Arbeitsplätzen gleichrangiges Ziel erscheint, und das allein in unserem Textkorpus 118 Mal. Dass Wachstum Arbeitsplätze schaffe(n könne), also ein Mittel von Politik in diesem Sinne sei, wird hingegen explizit nur an 13 Stellen gesagt.

Ein drittes sprachliches Muster affirmiert Wachstum dadurch als Politikziel, dass es als Bewertungskriterium bestimmter politischer Programme und Strategien erscheint. Wichtig sind hierfür Programme, die durch ihren bloßen Namen das Wachstumsziel „mitsetzen“, egal ob dieses dann separat noch einmal hervorgehoben wird; am prominentesten darunter der Europäische Stabilitäts- und Wachstumspakt und das in Abschn. 1 erwähnte Stabilitätsgesetz von 1967. Teilweise werden solche Programme auch dafür kritisiert, dass sie zwar Wachstum fördern sollen, dies aber nicht tun, wie im folgenden Beispiel aus einem Antrag der Linken für ein ,,Soziales Europa“:

[D]ie vorgeschlagenen und teils auf den Weg gebrachten Maßnahmen - Jugendbeschäftigungsinitiative und Jugendgarantie, die Pläne für ein europäisches Netz der Arbeitsvermittlungen (EURES) sowie der Pakt für Wachstum und Beschäftigung - [...] zielen einseitig darauf ab, die Mobilität von Arbeitnehmerinnen und Arbeitnehmer zu erhöhen, und forcieren damit die Abwanderung von Fachkräften anstatt durch gezielte Wirtschaftsförderung und öffentliche Investitionen - gerade in den sogenannten Krisenstaaten und strukturschwachen Regionen - Arbeitsplätze zu schaffen und Wachstumsimpulse zu setzen. $(18 / 1116)$

Dieses dritte Muster tritt deutlich seltener auf als die beiden vorgenannten; wie man aber aus seinem Bewertungscharakter unschwer ableiten kann, prägt es dafür auch Äußerungen der Opposition deutlich stärker mit. Exemplarisch dafür stehen auch zwei Plenardebatten um Griechenland und die Eurokrise (BT 18/117 und 18/118). Hier stellt die Generierung von Wachstum meist das oberste Bewertungskriterium für die Hilfsmaßnahmen dar, dem sich andere Aspekte wie die soziale Verträglichkeit unterordnen müssen. So merkt die Linke durch einen Zwischenruf zu den Ausführungen eines SPD-Redners korrigierend an, dass das Wachstum bereits vor dem Regierungsantritt von Alexis Tsipras um $25 \%$ gesunken sei, nicht erst danach. An einer anderen Stelle kritisiert ein Redner derselben Partei, dass die bisherige Kürzungspolitik nicht in der Lage sei, das Wachstum der griechischen 
Volkswirtschaft zu steigern. Wachstum als Politikziel wird dadurch jedes Mal impliziert. Dies ist eine Gemeinsamkeit aller drei sprachlichen Muster: Die Aussage „Wachstum ist unbezweifelbar ein Ziel jeder guten Politik“ scheint gerade deshalb so effektiv durch die verschiedenen Äußerungen reproduziert werden zu können, weil man es implizit (grammatisch) als Zweck positioniert. Argumentierendes ,Einhaken“ an dieser Stelle müsste die Debatte unterbrechen und gewissermaßen das Thema wechseln. Keinem Sprecher erscheint dies möglich.

Dass Wachstum ein Mittel zum Zweck sein kann, wird in unserem Sample hingegen kaum thematisiert. Über die Hälfte der Codings in dieser Dimension entfallen auf die Jahreswirtschaftsberichte der Bundesregierung und Gutachten der Wirtschaftsweisen, wo Wachstum in erster Linie als Mittel verstanden wird, um den Staatshaushalt $\mathrm{zu}$ stabilisieren und Arbeitsplätze zu sichern. Eine Problematisierung der Zweck-Mittel-Beziehung erfolgt auch hierbei fast nie. Ein weiteres Fünftel der entsprechenden Passagen entfällt auf die beiden erwähnten Plenardebatten zur Eurokrise. Die Mittel-Grammatik kann den dort vorherrschenden Selbstzweck-Attributionen natürlich schon quantitativ nicht Paroli bieten. Aber sie schöpft auch qualitativ ihr Potenzial zu einer stärkeren Problematisierung von Wachstum nicht aus. Eine genauere Argumentation zur Angemessenheit von Wachstum als (hinreichendes) Mittel z. B. zur Stabilisierung des Staatshaushalts wird interessanterweise dadurch verhindert, dass Wachstum als Glied einer Argumentationskette erscheint, in der z. B. das Griechenland-Hilfspaket als Mittel für Wachstum und dieses wiederum als Bedingung fiskalischer Konsolidierung fungiert - welche letztere dann öfter auch wiederum als Bedingung für weiteres Wachstum dargestellt wird. Diese Konstruktionen artikulieren eine Interdependenz von Wachstum und ausgeglichenem Haushalt, die tendenziell tautologische Züge aufweist. In den Plenardebatten springt diese Tendenz oft nicht so klar ins Auge, da sich die entsprechenden Passagen auf verschiedene Stellen der Beiträge verteilen. Evident aber wird die Tautologie in schriftlichen Äußerungen der Bundesregierung, etwa wenn es heißt:

Der gestärkte Stabilitäts- und Wachstumspakt bietet einen geeigneten Rahmen, um die Haushalte auf eine nachhaltig solide Basis zu stellen, die Schuldentragfähigkeit in allen Euroländern sicherzustellen und gleichzeitig wachstumsfreundlich Prioritäten so zu setzen, dass sich Fiskaldisziplin und Stärkung des Wachstums gegenseitig unterstützen. (18/3840)

Während solche Stellen ein argumentatives „Einhaken“ bezüglich der Funktionalität von Wachstum deshalb abweisen, weil sie schlicht verwirrend sind, wird in der mündlichen Rede die entsprechende Tautologie gelegentlich als Interdependenz anerkannt und dadurch entschärft; es heißt dann z. B. bei der Bundeskanzlerin, dass ,nachhaltiges Wachstum und solide Haushalte [einander] bedingen“ (Regierungserklärung vom 19. März 2015). In beiden Fällen aber wird der Charakter von Wachstum als Mittel im selben Moment, da er sichtbar wird, auch wieder relativiert, und Wachstum kann wieder als Politikziel erscheinen.

Die Aussage „Wachstum ist Politikziel“, die als Kurzfassung des in Abschn. 2 benannten Wachstumsparadigmas interpretiert werden kann, gewinnt also im Bundestag vor allem in Plenardebatten und Unterrichtungen durch die Bundesregierung eine diskursive Präsenz. Dass dabei Pro- oder Contra-Argumente qua Grammatik 
sich schlicht „nicht anbieten“, eines hohen Aufwandes seitens der Sprecher bedürften, kann man als Mechanismus begreifen, der die Aussage ,gegen empirische und terminologische Überprüfung abschottet" und damit im wörtlichen Sinne dogmatisch wirkt (Elze 1972, S. 277). Dieser Effekt wird im Diskurs außerdem durch ein rhetorisches Mittel verstärkt - worunter hier in einem engen Sinne das sprachliche Operieren nicht im Modus einer grammatischen Relation (sowie der von Zweck und Mittel), sondern durch semantische „Gleichsetzung“ verstanden wird (Burke 1978, S. 334-335). Solche Äquivalenzen pflegen im Fokus einer an Hegemonie interessierten Diskursanalyse zu stehen (Nonhoff 2010, S. 307). Implizite, metaphorische Gleichsetzungen, also etwa die der Wirtschaft mit einem Organismus durch die Rede von ,gesundem“ Wachstum usw., haben bei der Etablierung des Wachstumsdiskurses im Bundestag eine Rolle gespielt (Krohn 2007, S. 69), jedoch haben wir sie weniger häufig aufgefunden als explizite Synonyma. Die gedankliche Strukturleistung - Konzepte als etwas anderes zu verstehen - ist indes die gleiche wie bei Metaphern (Lakoff und Johnson 1980, S. 4-6).

Der Begriff, der am häufigsten mit Wachstum gleichgesetzt wird, ist „Entwicklung": so etwa, wenn im Fortschrittsbericht zur Energiewende (18/3487) direkt unter der Überschrift „Preiseffekte und Wachstumsimpulse“ davon die Rede ist, dass der Energiepreis „Einfluss auf die gesamtwirtschaftliche Entwicklung nehme“, oder wenn ein SPD-Abgeordneter in der Sondersitzung zu Griechenland affirmiert: „Ohne wirtschaftliche Entwicklung, ohne Wachstum ist jede Schuldenfähigkeitsdiskussion hinfällig." Die syntaktischen Mittel der Gleichsetzung variieren, der Effekt ist jedoch unzweideutig: Wachstum und Entwicklung sind dasselbe. In weniger als einem Drittel der Fälle kommt ein metaphorischer Überschuss im Sinne der Krohnschen Analyse hinzu, nämlich wenn von „Erholung“ oder „Belebung“ der Wirtschaft die Rede ist.

Ebenfalls präsent sind Gleichsetzungen von Wachstum mit Wohlfahrt oder „,Wohlstand": Sie finden sich in den Jahreswirtschaftsberichten, in den daran anschließenden Regierungserklärungen des Ministers, im Jahresgutachten des Sachverständigenrates, aber rudimentär auch in der TTIP-Debatte, bei der sowohl Die Linke als auch die ihr antwortende Bundesregierung von erwarteten „Wohlfahrtseffekten“ sprechen und Wachstumsprognosen meinen. Die zugrundeliegende Auffassung war nicht nur von der Enquete-Kommission ausdrücklich zurückgewiesen worden, indem sie „materielle“ Wohlstandsindikatoren von ökologischen Indikatoren und solchen der „Teilhabe“ begrifflich schied (Deutscher Bundestag 2013, S. 233-238). Auch in unseren MdB-Interviews haben wir differenzierte Wohlstandsbegriffe gefunden, in denen materielle Komponenten durchaus eine Rolle spielten, aber nicht durchweg zentral waren (Rivera et al. 2016, S. 22). Der Diskurs der Dokumente ist hier also erneut indifferent oder äußerlich gegenüber möglichen Argumenten der ihn tragenden Akteure; womit wir auf Foucaults Exterioritätsprinzip stoßen (siehe Abschn. 2).

Neben Gleichsetzungen von Wachstum mit anderen Hochwertbegriffen, also einer offensiven Rhetorik, findet sich ein eher unterschwelliges Muster der Verbreitung, nämlich die Durchsetzung der Texte mit technischen Angaben. Dies betrifft knapp zehn Prozent aller Codings; sie verknüpfen das BIP - also einen integralen Bestandteil des Wachstumsparadigmas - mit einer Reihe von anderen Maßgrößen der Wirtschaft. Zu diesen technischen Angaben zählen etwa die Arbeits-, Energie- 
oder Rohstoffproduktivität, die Lohn- oder die Sparquote; vor allem jedoch Angaben, welche die Staatsfinanzen betreffen, wie etwa der Primärsaldo oder die Schuldenstandsquote. Die entsprechende Technikprosa erscheint gegenüber möglichen Wachstumsaussagen zwar argumentativ neutral, lässt jedoch ihre Referenzgröße die durch das BIP gemessene Wirtschaftsleistung - als gewissermaßen unersetzlich erscheinen. Auch hier handelt es sich also um einen Parameter, der zumindest indirekt zur Reproduktion einer Art Dogma mit beiträgt.

\section{Ansätze zur Relativierung oder Verteidigung von Wachstum}

Wie wir sahen, wird der Wachstumsbegriff vor allem von den Koalitionsfraktionen genutzt, und zurückhaltender von denen der Opposition. Das wird auch durch die Analyse Großer und Kleiner Anfragen bestätigt, die wichtige Kontroll- und Kommunikationsinstrumente der Opposition darstellen, in denen Wachstum aber nur in geringem Umfang thematisiert wird. So wurde in den neun Großen Anfragen, die innerhalb des Untersuchungszeitraums gestellt wurden, nur in einer Anfrage der Linken zu den Effekten des geplanten Freihandelsabkommens zwischen der EU und den USA (TTIP), Wachstum überhaupt angesprochen, und dies auch nur in geringem Umfang (sowie seinen Status als Politikziel affirmierend) (BT 18/432). Ähnliches gilt für die Kleinen Anfragen, welche ebenfalls als wichtiges Instrument der Regierungskontrolle gelten (Ismayr 2012, S. 326-330; Kepplinger 2007, S. 308). Die Dokumente werden vielfach weiterverbreitet und dienen zum einen als Bezugspunkt für Stakeholder (Siefken 2010, S. 27, 31-33) und zum anderen der Kommunikation zwischen Parteibasis und Abgeordneten (Ismayr 2012, S. 328). Von den im Untersuchungszeitraum eingereichten Kleinen Anfragen enthielten weniger als ein Prozent den Begriff Wachstum im Sinne unseres Forschungsinteresses. Die Analyse verdeutlicht, dass die oppositionellen Parteien zwar weniger an der Reproduktion des Wachstumsdogmas beteiligt sind; sie versuchen aber auch nicht, es aufzubrechen. So waren kaum ein Viertel der Referenzen in den Kleinen Anfragen wachstumsrelativierend oder gar -kritisch. Offenbar sind die Oppositionsparteien nicht übermäßig daran interessiert, die Thematik in den Bundestag hineinzutragen und dabei mit wachstumskritischen Stakeholdern in Verbindung zu treten.

Dabei zeigen sich freilich Unterschiede zwischen den beiden Oppositionsfraktionen. So taten sich Bündnis90/Die Grünen beim Obercode ,Wachstum ist nicht alles“ deutlich hervor. Ein Drittel aller Codings dieser Fraktion war unter diese Kategorie zu subsumieren, mit Tendenzen einerseits zu einer Separierung des Wohlstandsvom Wachstumsbegriffs, andererseits zur Hervorhebung ,bedingten“, insbesondere „grünen“ Wachstums (Green Growth). Insgesamt thematisieren grüne Abgeordnete Wachstum deutlich häufiger als die Abgeordneten der Linken. Besonders deutlich wird der Unterschied in den Anträgen der Fraktionen. In denen der Grünen fanden wir fast dreimal so viele relevante Sinneinheiten wie in denen der Linken. Hierzu passt, dass Wachstum innerhalb dieser Partei auch Gegenstand von Diskussionen ist. In unseren Leitfaden-Interviews berichteten alle Vertreterinnen von Bündnis 90/Die Grünen - aber kein einziger einer anderen Partei - es gebe eine entsprechende De- 
batte in ihrer Fraktion. Aus der Perspektive einer bekennenden Wachstumskritikerin beschrieben:

Wir haben seit über einem Jahr jetzt eine Fraktionsarbeitsgruppe zu dem Thema „ökologische soziale Transformation der Wirtschaft“, wo zwei, drei andere Kollegen und ich explizit auch für die Frage von Postwachstumsdenken, DeGrowth-Gedanken und ähnlichen Dingen auch stehen. [...] Da gibt es natürlich noch großen Klärungsbedarf auch innerhalb der Fraktion, ob man eigentlich das Wachstumsmodell insgesamt infrage stellt oder ob man nur sagt: Man stellt das Wirtschaftsmodell infrage, aber man geht dann trotzdem davon aus, dass es weiteres Wachstum geben wird - oder eben nicht.

Der Umstand, dass bei den Grünen Wachstum ein Thema ist, über das die einzelne Abgeordnete sich politisch positionieren und profilieren kann, mag erklären, warum in den von ihnen verantworteten Diskursbeiträgen sogar genuine Wachstumskritik zumindest auftaucht. ${ }^{12}$ Diese Kritik richtet sich entweder gegen ein bestimmtes nicht wünschenswertes Wachstum, wie „Wirtschaften auf Kosten von Mensch, Natur und Umwelt“. Oder das BIP als Wohlstandsmaß wird generell infrage gestellt und die Einführung anderer Indikatoren gefordert, wie im Antrag zur Einführung eines Jahreswohlstandberichts, der auch explizit an die Enquete anschließt (18/7368). Dass solche Argumente vor allem durch die Grünen getragen werden, belegt im größeren Blick auf das Gesamtspektrum des Parlamentsdiskurses noch lange nicht, dass die Wachstumsdebatte ,distinktiv“ für diese Partei ist; zumindest legt ein korpuslinguistischer Blick auf die 11.-17. Legislaturperiode dies nicht nahe (Heiberger und Koss 2018, S. 411-413). Es markiert indessen immerhin den Ansatz einer diskursiven Differenzierung, die auf Positionen von Akteuren beziehbar ist.

Während Wachstumskritik sich bei den Koalitionsfraktionen niemals findet, teilen sie mit der Opposition doch gewisse argumentative Muster, um Wachstum zu relativieren. Ein Weg, dies zu tun, ist das Knüpfen an Bedingungen: Nur einige, ,qualitativ“ bestimmte Arten von Wachstum seien gut für die Gesellschaft. Dies stellt keine Ablehnung des Wachstumsparadigmas dar, denn das „qualitative“ Wachstum muss nicht limitiert werden. Eine Ausnahme bildet die Qualifizierung, die Wachstum klar nur innerhalb ökologischer Grenzen erlauben will; sie erscheint jedoch nur sehr selten. Am häufigsten wird hingegen auf ,nachhaltiges Wachstum“ verwiesen. Die unklare Definition dieses Begriffs ermöglicht, dass er in sehr unterschiedlichen Kontexten verwendet wird. Einmal wird damit Wachstum beschrieben, das Ressourcen auch im Hinblick auf zukünftige Generationen schont. In diesem Sinne sind Verweise auf die Europa-2020-Strategie gefasst, die ,intelligentes, integratives und nachhaltiges Wachstum“" schaffen will, sowie Bezüge zum Ziel 8 der UNNachhaltigkeitsagenda 2030. Daneben findet sich auch eine andere Verwendung, bei der nachhaltiges Wachstum im Kontext von Schuldenabbau und Haushaltskonsoli-

\footnotetext{
12 Ausgehen können wir aufgrund unserer Daten, wie in Abschn. 1 bereits erwähnt, von einer beträchtlichen Meinungsvielfalt und Offenheit gegenüber Debatten bei den Abgeordneten und ihren Mitarbeitern. So stimmten in unserer repräsentativen Erhebung z. B. $40 \%$ der Mitarbeiter der Aussage, ,Wenn die Wirtschaft wächst, dann geht es uns allen besser“, eher nicht oder gar nicht zu; ein Drittel der Befragten teilten sogar eher bzw. voll und ganz eine klar formulierte Wachstumskritik (,Wenn die Wirtschaft wächst, dann geht es uns allen besser“). Weitere Details hierzu in Rivera et al. (2016), S. 20-30.
} 
dierung problematisiert wird. Hier bezieht sich die Nachhaltigkeit des Wachstums eher auf ein stetiges Wachstum, das nicht durch die Aufnahme neuer Schulden generiert wird (z.B. BT 18/117; 18/60). Diese Verwendung findet sich vor allem in Regierungserklärungen und Plenardebatten (mit Bezug zu Griechenland und der Eurokrise), also bei Sprechakten, die direkt an eine breitere Öffentlichkeit adressiert sind. Die von Anfang an diffuse Bedeutung der Formel ,nachhaltiges Wachstum“, bei ihrer Prägung im 14. Deutschen Bundestag wohl ein Versuch der rotgrünen Koalition, ,,sprachlich eine Zielharmonie von Wachstum und Umweltschutz auszudrücken“ bzw. zu simulieren (Krohn 2007, S. 72), ist also in den vergangenen Legislaturperioden weiter aufgeweicht. Man kann darin einen Beleg mehr für die schon oft beschriebene diskursstabilisierende Kraft des „leeren Signifikanten“ Nachhaltigkeit sehen (Kronfeld-Goharani 2015, S. 317-322), und zwar erneut über verschiedenste Politikbereiche und Fraktionen hinweg.

Jeweils (fast) ebenso häufig wie das ,nachhaltige Wachstum“ werden etwa qualifizierende Frames genutzt, die in der OECD und anderen internationalen Diskursen in Reaktion auf die Wachstumskritik generiert und verbreitet wurden (Schmelzer 2015, S. 268-269): Smart Growth (die Digitalisierung begünstigt die Ausweitung des Humankapitals und/oder eine Reduktion der Materialen, die in der Produktion benötigt werden); Green Growth (bestimmte Technologien, wie die Erneuerbaren Energien, ermöglichen Wachstum, das der Umwelt nicht schadet); Inclusive Growth (Wachstum, das sowohl Armut als auch Ungleichheit reduziert und von dem die ärmsten Mitglieder der Gesellschaft profitieren). Tendenziell sind diese Frames eher gouvernemental; die Green-Growth-Attributionen erfreuen sich jedoch auch bei den Grünen gewisser Beliebtheit. Die Frames wirken als Abkürzungen oder Signa der in Klammern kurz angedeuteten Storylines; um diese ,,aufzurufen“, genügt ein Teilelement (Hajer 1995, S. 62-63; siehe auch Fußnote 8).

Das Wachstum „qualifizierende“ Formulierungen sind nicht sosehr deshalb interessant, weil sie im Diskurs eine argumentative Auseinandersetzung ermöglichten - dies tun sie nur höchst bedingt. Als vermittelnde Positionen sind sie jedoch das Ergebnis, die diskursive Spur einer reflexiven, argumentativen Reaktion auf Wachstumskritik: entweder auf die Frage nach der Machbarkeit von Wirtschaftswachstum (Smart Growth und Green Growth) oder jener nach der Wünschbarkeit (Inclusive Growth). Den Verteidigungen von Machbarkeit liegt dabei ein gemeinsamer Innovationsframe zugrunde: Technologische Erfinderkraft lässt uns die Grenzen des Wachstums immer wieder hinausschieben, indem wir neue Ressourcen erschließen, alte substituieren usf. Dieser wirtschaftswissenschaftlich inspirierte Frame hat Knauß' Medienanalyse zufolge in den Siebzigerjahren in Reaktion auf die Wachstumsskepsis des Club of Rome zunehmende gesellschaftliche Verbreitung gefunden, um spätestens in der 15. Legislaturperiode auch in Teilen der Grünen Partei feste Wurzeln zu schlagen (Knauß 2016, S. 135-140). In unserer Analyse hingegen fällt auf, dass zwar sein Hauptmotiv - die Wichtigkeit von Innovationen - im Bundestagsdiskurs relativ präsent ist, es jedoch in den von uns untersuchten Dokumenten niemals explizit dazu verwendet wird, Wachstumsgrenzen zu relativieren. Die schiere Dogmatik und Ornamentalität des Diskurses, so können wir spekulieren, machen diese explizite Verteidigungsstrategie wohl schlicht entbehrlich. 
Etwas anders sieht es mit dem Standortframe aus, der die Sorge um ausbleibendes Wachstum als Befürchtung ,kollektiven Niedergangs“ artikuliert (Knauß 2016, S. 144). Sieht Knauß es in den Medien auf dem Rückzug, seit Deutschlands Wachstumsraten sich wieder stabilisierten, finden wir es im Bundestag im Vergleich zu anderen Frames überraschend häufig (48 Mal in unserem Korpus). Inhaltlich ist daran bemerkenswert, dass der Standortframe im Unterschied zum Innovationsframe nicht die Machbarkeit fortwährenden Wachstums verteidigt, sondern nur seine Wünschbarkeit (indirekt) affirmiert. Diskursiv wird über den ,,Standort Deutschland“ ein Kollektivakteur als Subjektposition angeboten, der eine in der parlamentarischen Arena möglicherweise zugkräftigere Aussage impliziert als der menschheitsoder wissenschaftsbezogene Innovationsframe: Deutschland braucht Wachstum, um sich gegenüber anderen Nationen behaupten zu können. Die entsprechende Rede von der Wettbewerbsfähigkeit (meist Deutschlands, seltener Europas) kennzeichnet denn auch über die Hälfte aller Fundstellen des Standortframes; Wachstum wird dabei wiederum kein Thema, sondern wird als Politikziel (sehr indirekt) impliziert.

\section{Fazit und Ausblick}

Wenn eine dogmatische Schließung Debatten über eine gesellschaftlich durchaus virulente politische Grundsatzfrage im Parlament verhindert, dann ist dies, wie bereits in der Einleitung bemerkt, ein Problem nicht nur für die demokratische Bearbeitung der jeweiligen Frage, sondern für die Erfüllung der Öffentlichkeitsfunktion des Parlaments, gleich ob man dabei den „Stil“" der Parlamentarierkommunikation als eher „responsiv“ oder ,orientierend“ begreift. ${ }^{13}$ Im Falle der Grundsatzfrage Wirtschaftswachstum haben wir gesehen, wie in den verschiedenen Teilarenen des Bundestags eine argumentative Thematisierung ausbleibt, obwohl wir andererseits klare Hinweise auf stark variierende Einstellungen der MdBs und ihrer Mitarbeiterinnen haben. Letztere finden sich nicht nur in unserer Erhebung, sondern schon in früheren Studien, wobei dort ebenfalls zu beobachten ist, dass diejenigen MdBs mit längerer Verweildauer im Parlament das Wachstum höher priorisieren (Best und Vogel 2012, S. 42-43) - ein ,hintergrundunabhängiger parlamentarischer Sozialisationseffekt", der auch bei anderen Fragestellungen insofern beobachtbar ist, dass die Salienz von Themen sich angleicht bzw. Unterschiede zwischen den Äußerungen verschiedener Abgeordneter sich einebnen (am Beispiel von Migrationsthemen Blätte und Wüst 2017, S. 212, 229; für verschiedene steuerpolitische Frames Heinemann und Janeba 2010, S. 299). Der oberflächliche Eindruck, dass im Fall der Frage Wirtschaftswachstum der Effekt darin besteht, dass die Abgeordneten in eine Art Litanei eingeübt werden, verdichtet sich angesichts der Analyse des öffentlichen Diskurses, wie wir sie hier vorgelegt haben, zur Gewissheit. Besonders bemerkenswert ist dabei, dass der dogmatische Kern dieser Litanei (Wirtschaftswachstum als Selbstzweck) von einem vorhergehenden innerparlamentarischen Lernprozess (der

\footnotetext{
13 MdBs tun dies in insgesamt eher ausgewogener Form, mit einer gewissen Bevorzugung des ,orientierungbietenden“ Rollenverständnisses; nur bei weiblichen und vor allem oppositionellen Abgeordneten verschieben sich die Gewichtungen hin zur Responsivität (Best et al. 2007, S. 11).
} 
Enquete-Kommission der 17. Legislaturperiode) vollkommen unberührt geblieben ist, und dies, obwohl der oft genannte Haupt-Obstruktor dieses Prozesses, die FDPFraktion, im Untersuchungszeitraum am Bundestagsdiskurs nicht beteiligt war.

$\mathrm{Ob}$ dieser Befund Entsprechungen bei anderen Grundsatzfragen hat - bei solchen, die etabliert, vor allem aber bei solchen, die ,im Kommen“ sind - ist nachprüfenswert, wenn man sich für die Reflexivität demokratischer Institutionen interessiert. Dabei ist u. U. erwart- und nachvollziehbar, dass die Grundsatzfragen im Herzen der gesetzgeberischen Aktivität selber nicht nochmals erörtert werden (vgl. Abschn. 3). Sollten die mit ihnen zusammenhängenden Begriffe indes, wie an unserem Beispiel zu beobachten, auch in anderen Teilarenen des Parlaments auf den Status des „Phrasenschweins“ oder zumindest der extremen Formelhaftigkeit degradiert werden, dann wäre dies aus einer Teilnehmerperspektive heraus zunächst einmal bedenklich. Solche Phänomene gäben alten antiparlamentarischen Vorurteilen (,Schwatzbude“ etc.) neue Nahrung. Insbesondere das Plenum als Darstellungsoberfläche des Parlaments bietet, sofern ein solcher begrifflicher Ritualismus Raum greift, hierfür Angriffspunkte, die durch den bloßen Verweis auf den Arbeitscharakter der Gesamtinstitution nicht unbedingt ausgeräumt werden (Willemsen 2015, S. 112-113, 401-403). Die denkbare Gegenhypothese, dass das Wachstumsdogma lediglich ein diskursiver Sonderfall ist, z.B. durch strukturelle Zwänge des Sozialstaats bedingt (Zahrnt und Seidl 2012), wäre zu untersuchen.

Forschungspragmatisch noch interessanter als solche einem normativ-demokratietheoretischen Interesse an Responsivität verpflichteten Überlegungen ist indes möglicherweise - der durch uns gewissermaßen à contrecœur vollzogene Halbschritt zu einem eher poststrukturalistischen Verständnis des politischen Diskurses selbst. Er deutet darauf hin, dass Jenni Brichzin und ihre Mitautoren recht haben, wenn sie die Arbeitsteilung zwischen einer auf Mikro- und Mesoprozesse (z. B. Rollenhandeln, Principal-Agent-Verhältnisse) verpflichteten Soziologie und einer stärker Makrostrukturen (Stellung im politischen System) zugewandten Politikwissenschaft für die Parlamentsforschung als veraltet zurückweisen und statt dessen darauf insistieren, dass Sozialtheorien - unter ihnen auch poststrukturalistische Diskurstheorien - bei empirischen Analysen des Verhältnisses von Parlament und Gesellschaft stärker und vielfältiger (über den lange dominierenden Strukturfunktionalismus hinaus) einbezogen werden müssen (Brichzin et al. 2018, S. 16-17). Dabei ist „Responsivität" dann möglicherweise nur noch ein Sonderfall jenes Verhältnisses, scharf gestellt durch die demokratietheoretische Brille (Brichzin et al. 2018, S. 10-11). Das Verhältnis des Parlaments zur Öffentlichkeit aber ist ganz offenbar auch noch durch andere Mechanismen als solche des Verhältnisses von „Bürgern“ und „Repräsentanten“ mitgeprägt. Diese (auch) diskursanalytisch zu untersuchen, empfiehlt sich gerade beim Interesse für langfristige Prozessverläufe und Themenkonjunkturen (Heiberger und Koss 2018), wobei die Einsicht in ein „Teilhaben“ der Parlamentarier an übergreifenden Diskursen sie vor überzogenen Erwartungen an ihre Autonomie (Patzelt 1998) u. U. sogar bewahren kann.

Je größer die im Zeitalter der Digitalisierung verfügbar werdenden Analysekorpora, desto näher liegt der Rückgriff auf quantitative Methoden der Korpuslinguistik. Ohne deren Nutzen in Abrede zu stellen, können wir anhand der vorliegenden Untersuchung doch auch auf die Vorteile eines mit quantifizierenden Elementen 
angereicherten qualitativen Ansatzes verweisen, der es ermöglicht, wichtigen Fragen nachzugehen, die über die Semantik nicht abbildbar sind. Zur ,,allmähliche[n] Errichtung einer abstrahierenden Analyseebene" ist eine solche Nähe zum offenen Kodieren auch im Bereich poststrukturalistisch orientierter Diskursanalyse anerkannt und, in Kombination mit Sequenzanalysen, fruchtbar gemacht worden (DiazBone 2002, S. 199; Diaz-Bone und Schneider 2010, S. 514). Wir möchten jedoch insbesondere darauf abheben, dass grammatische Strukturmerkmale der Rede im Bereich mittelgroßer Korpora halbsatzgenau rekonstruiert, aber auch quantitativ so hoch aggregiert werden können, dass textübergreifende Merkmale jenseits des Sinns einzelner Argumente beschreibbar werden. In unserem Falle war dies das - freilich zunächst deduktiv gesetzte - Verhältnis von politischem Zweck und Mittel. Wir konnten plausibel machen, dass implizite Setzungen von Begriffen oder Programmen - hier: dem Wachstumsparadigma - als Zweck ein diskursiver Mechanismus sein könnten, die Thematisierung oder Erörterung dieses Zweckes unwahrscheinlich zu machen.

Letzteres ist für Diskursanalysen auch deshalb eine interessante Fährte, weil die spätestens seit Max Weber im Raum stehende sozialtheoretische Frage, ob eine höhere „Wertrationalität“ im Sinne „,bewusste[re]n Glaubens““ an bzw. einer Verständigung über kollektive Ziele in der Moderne überhaupt möglich ist (Weber 2005, S. 17-18; Habermas 1995, S. 377-385), im Bereich der Politik eine besondere normative Schärfe erhält - siehe hierzu auch das Eröffnungszitat dieses Aufsatzes. Inwiefern dabei dem ,Implizieren“ von Zielsetzungen, ihrer eigentümlich verschleiernden Affirmation, eine obstruktive Rolle zukommt, und ob es vielleicht noch ganz andere diskursive Mechanismen des Ins-Verhältnis-Setzens von Zweck und Mittel gibt, ist intensiverer Untersuchungen wert.

Open Access Dieser Artikel wird unter der Creative Commons Namensnennung 4.0 International Lizenz (http://creativecommons.org/licenses/by/4.0/deed.de) veröffentlicht, welche die Nutzung, Vervielfältigung, Bearbeitung, Verbreitung und Wiedergabe in jeglichem Medium und Format erlaubt, sofern Sie den/die ursprünglichen Autor(en) und die Quelle ordnungsgemäß nennen, einen Link zur Creative Commons Lizenz beifügen und angeben, ob Änderungen vorgenommen wurden.

\section{Literatur}

Best, Heinrich, und Lars Vogel. 2012. The emergence and transformation of representative roles. In Parliamentary roles in modern legislatures, Hrsg. Magnus Blomgren, Oliver Rozenberg, 37-65. London: Routledge.

Best, Heinrich, Michael Edinger, Karl Schmitt, und Lars Vogel. 2007. Zweite Deutsche Abgeordnetenbefragung 2007: Dokumentation für den Deutschen Bundestag. Jena: Friedrich-Schiller-Universität.

Best, Heinrich, Michael Edinger, Daniel Gerstenhauer, und Lars Vogel. 2010. Jenaer Parlamentarierbefragung 2010: Dokumentation zum Deutschen Bundestag. Jena: Friedrich-Schiller-Universität.

Blätte, Andreas, und Andreas M. Wüst. 2017. Der migrationsspezifische Einfluss auf parlamentarisches Handeln: Ein Hypothesentest auf der Grundlage von Redebeiträgen der Abgeordneten des Deutschen Bundestags 1996-2013. Politische Vierteljahresschrift 58(2):205-233.

Bohrmann, Ulf, und Henning Laux. 2018. Das synchronisierte Parlament - eine differenzierungstheoretische Perspektive. In Soziologie der Parlamente, Hrsg. Jenni Brichzin, Damien Krichewsky, Leopold Ringel, und Jan Schank, 287-305. Wiesbaden: Springer VS.

Brand, Ulrich. 2014. Degrowth: Der Beginn einer Bewegung? Blätter für deutsche und internationale Politik 10:29-32.

Brichzin, Jenni, Damien Krichewsky, Leopold Ringel, und Jan Schank. 2018. Soziologie der Parlamente. Konturen eines Programms. In Soziologie der Parlamente, Hrsg. Jenni Brichzin, Damien Krichewsky, Leopold Ringel, und Jan Schank, 1-32. Wiesbaden: Springer VS. 
Bröckling, Ulrich, Susanne Krassmann, und Thomas Lemke (Hrsg.). 2000. Gouvernmentalität der Gegenwart. Frankfurt: Suhrkamp.

BUND, und Brot für die Welt. 2010. Zukunftsfähiges Deutschland in einer globalisierten Welt. Ein Anstoß zur gesellschaftlichen Debatte. Frankfurt: Fischer.

BUND, und Misereor. 1997. Zukunftsfähiges Deutschland. Ein Beitrag zu einer global nachhaltigen Entwicklung, 4. Aufl., Basel: Springer.

Burke, Kenneth. 1968. Dramatistic Method. In International Encyclopedia of the Social Sciences, Bd. 7 , Hrsg. D.L. Sills, 445-447. New York: Macmillan.

Burke, Kenneth. 1978. Questions and answers about the pentad. College Composition and Communication 29(4):330-335.

Busse, Dietrich, und Wolfgang Teubert. 1994. Ist Diskurs ein sprachwissenschaftliches Objekt? Zur Methodenfrage der Historischen Semantik. In Begriffsgeschichte und Diskursgeschichte, Hrsg. Dietrich Busse, Fritz Herrmanns, und Wolfgang Teubert, 10-28. Opladen: Westdeutscher Verlag.

Daly, Herman E. 1993. Steady-state economics: a new paradigm. New Literary History 24(4):811-816.

Deutscher Bundestag. 1967. Gesetz zur Förderung der Stabilität und des Wachstums der Wirtschaft $(\operatorname{StabG})$. Bonn: Deutscher Bundestag.

Deutscher Bundestag. 2013. Schlussbericht der Enquete-Kommission „Wachstum, Wohlstand, Lebensqualität - Wege zu nachhaltigem Wirtschaften und gesellschaftlichem Fortschritt in der Sozialen Marktwirtschaft". Bonn: bpb.

Diaz-Bone, Rainer. 2002. Kulturwelt, Diskurs und Lebensstil. Eine diskurstheoretische Erweiterung der bourdieuschen Distinktionstheorie. Opladen: Leske + Budrich.

Diaz-Bone, Rainer, und Werner Schneider. 2010. Qualitative Datenanalysesoftware in der sozialwissenschaftlichen Diskursanalyse - zwei Praxisbeispiele. In Forschungspraxis Handbuch Sozialwissenschaftliche Diskursanalyse, Bd. 2, Hrsg. Rainer Keller, Andreas Hirseland, Wolfgang Schneider, und Willy Viehöver, 491-529. Wiesbaden: VS.

Elze, Martin. 1972. Dogma. In Historisches Wörterbuch der Philosophie, Bd. 2, Hrsg. Joachim Ritter, 275-277. Basel: Schwabe.

Foucault, Michel. 1972. The archaeology of knowledge and the discourse on language. New York: Pantheon Books.

Früh, Werner. 2007. Inhaltsanalyse, 6. Aufl., Konstanz: UVK.

Gerhards, Jürgen. 2010. Systematische Inhaltsanalyse - Öffentliche Abtreibungsdiskurse. In Forschungspraxis, 4. Aufl., Handbuch Sozialwissenschaftliche Diskursanalyse, Bd. 2, Hrsg. Rainer Keller, Andreas Hirseland, Wolfgang Schneider, und Willy Viehöver, 333-358. Wiesbaden: VS.

Gerhards, Jürgen, und Friedhelm Neidhardt. 1993. Strukturen und Funktionen moderner Öffentlichkeit: Fragestellungen und Ansätze. In Politische Kommunikation. Grundlagen, Strukturen, Prozesse, Hrsg. Wolfgang Langenbucher, 52-89. Wien: Braumüller.

Habermas, Jürgen. 1995. Theorie des kommunikativen Handelns. Frankfurt: Suhrkamp.

Hajer, Maarten A. 1995. The politics of environmental discourse. Ecological modernization and the policy process. New York: Oxford University Press.

Hampel, Frank. 1991. Politikberatung in der Bundesrepublik: Überlegungen am Beispiel von EnqueteKommissionen. Zeitschrift für Parlamentsfragen 22(1):111-133.

Heiberger, Raphael H., und Christian Koss. 2018. Computerlinguistische Textanalyse und Debatten im Parlament. In Soziologie der Parlamente, Hrsg. Jenni Brichzin, Damien Krichewsky, Leopold Ringel, und Jan Schank, 391-418. Wiesbaden: Springer VS.

Heinemann, Friedrich, und Eckhard Janeba. 2010. Viewing tax policy through party-colored glasses: what German politicians believe. German Economic Review 12(3):283-311.

Ismayr, Wolfgang. 2012. Der Deutsche Bundestag, 3. Aufl., Wiesbaden: VS.

Jung, Matthias. 2011. Diskurshistorische Analyse - eine linguistische Perspektive. In Theorien und Methoden, 3. Aufl., Handbuch Sozialwissenschaftliche Diskursanalyse, Bd. 1, Hrsg. Reiner Keller, Andreas Hirseland, Wolfgang Schneider, und Willy Viehöver, 35-59. Wiesbaden: VS.

Kepplinger, Hans Mathias. 2007. Kleine Anfragen: Funktionale Analyse einer parlamentarischen Praxis. In Res publica semper reformanda. Wissenschaft und politische Bildung im Dienste des Gemeinwohls. Festschrift für Heinrich Oberreuter, Hrsg. Werner J. Patzelt, Martin Sebaldt, und Uwe Kranenpohl, 304-319. Wiesbaden: VS.

Kerchner, Brigitte. 2006. Diskursanalyse in der Politikwissenschaft. Ein Forschungsüberblick. In Foucault: Diskursanalyse der Politik. Eine Einführung, Hrsg. Brigitte Kerchner, Silke Schneider, 33-67. Wiesbaden: VS.

Knauß, Ferdinand. 2016. Wachstum über alles? Wie der Journalismus zum Sprachrohr der Ökonomen wurde. München: oekom. 
Krohn, Philipp. 2007. Ausweg Wachstum? Sprache in einer begrenzten Welt. In Ausweg Wachstum? Arbeit, Technik und Nachhaltigkeit in einer begrenzten Welt, Hrsg. Deutscher Studienpreis, 63-78. Wiesbaden: VS.

Kronfeld-Goharani, Ulrike. 2015. The discursive constitution of ocean sustainability. Advances in Applied Sociology 5:306-330.

Laclau, Ernesto, und Chantal Mouffe. 1991. Hegemonie und radikale Demokratie. Zur Dekonstruktion des Marxismus. Wien: Passagen Verlag.

Lakoff, George, und Mark Johnson. 1980. Metaphors we live by. Chicago: University of Chicago Press.

Lakoff, George. 2008. The political mind. A cognitive scientist's guide to your brain and its politics. New York: Penguin Books.

Link, Jürgen. 1983. Was ist und was bringt Diskurstaktik. kultuRRevolution 2:60-66.

Mayring, Philipp. 2010. Qualitative Inhaltsanalyse, 11. Aufl., Weinheim, Basel: Beltz.

Nonhoff, Martin. 2010. Hegemonieanalyse: Theorie, Methode und Forschungspraxis. In Forschungspraxis, 4. Aufl., Handbuch Sozialwissenschaftliche Diskursanalyse, Bd. 2, Hrsg. Rainer Keller, Andreas Hirseland, Wolfgang Schneider, und Willy Viehöver, 299-331. Wiesbaden: VS.

Novalis. 1984. Politische Aphorismen. In Fragmente und Studien, Hrsg. Carl Paschek, 59-64. Stuttgart: Reclam.

Nützenadel, Alexander. 2002. Wissenschaftliche Politikberatung in der Bundesrepublik. Die Gründung des Sachverständigenrates zur Begutachtung der gesamtwirtschaftlichen Lage 1963. Vierteljahresschrift für Sozial- und Wirtschaftsgeschichte 89(3):288-306.

von Oertzen, Jürgen. 2006. Das Expertenparlament. Abgeordnetenrollen in den Fachstrukturen bundesdeutscher Parlamente. Baden-Baden: Nomos.

Palfner, Sonja. 2006. Werkzeug Aussage - ein politikwissenschaftlicher Versuch. In Foucault: Diskursanalyse der Politik. Eine Einführung, Hrsg. Brigitte Kerchner, Silke Schneider, 210-230. Wiesbaden: VS.

Patzelt, Werner. 1998. Ein latenter Verfassungskonflikt? Die Deutschen und ihr parlamentarisches Regierungssystem. Politische Vierteljahresschrift 39(4):725-757.

Pennekamp, Johannes. 2011. Wohlstand ohne Wachstum. Ein Literaturüberblick. MPIfG Working Paper 11 /1. Köln: Max-Planck-Institut für Gesellschaftsforschung.

Rivera, Manuel. 2018. Growth in parliament: Some notes on the reproduction of a dogma. Futures 95:1-10.

Rivera, Manuel, Claudia Saalbach, Franziska Zucher, und Moritz Mues. 2016. Das Wachstumsparadigma im Deutschen Bundestag. Ergebnisse und Fragen aus dem Projekt „Growth in Politics“. IASS Study. Potsdam: IASS.

Schmelzer, Matthias. 2015. The growth paradigm: History, hegemony, and the contested making of economic growthmanship. Ecological Economics 118:262-271.

Schwab-Trapp, Michael. 2010. Methodische Aspekte der Diskursanalyse. Probleme der Analyse diskursiver Auseinandersetzungen am Beispiel der deutschen Diskussion über den Kosovokrieg. In Forschungspraxis, 4. Aufl., Handbuch Sozialwissenschaftliche Diskursanalyse, Bd. 2, Hrsg. Rainer Keller, Andreas Hirseland, Wolfgang Schneider, und Willy Viehöver, 171-196. Wiesbaden: VS.

Schwab-Trapp, Michael. 2011. Diskurs als soziologisches Konzept. Bausteine für eine soziologisch orientierte Diskursanalyse. In Theorien und Methoden, 3. Aufl., Handbuch Sozialwissenschaftliche Diskursanalyse, Bd. 1, Hrsg. Reiner Keller, Andreas Hirseland, Wolfgang Schneider, und Willy Viehöver, 283-307. Wiesbaden: VS.

Siefken, Sven T. 2010. Parlamentarische Frageverfahren - Symbolpolitik oder wirksames Kontrollinstrument? Zeitschrift für Parlamentsfragen 41(1):18-36.

Ueberhorst, Reinhard. 2014. Über den Umgang mit nicht beliebigen kooperativen Leistungszielen im Arbeitsprozess der wachstumspolitischen Enquete-Kommission des Deutschen Bundestages (2011-2013). In Forschung für die Wirtschaft 2013, Hrsg. Georg Plate, 315-342. Göttingen: Cuvillier.

Vogel, Lars. 2014. Sonderauswertung MdB der Jenaer Parlamentarierbefragung für das IASS. Jena: Universität. Unveröffentlicht.

Weber, Max. 2005. Wirtschaft und Gesellschaft. Frankfurt: Zweitausendeins.

Wengeler, Martin, und Alexander Ziem. 2010. „Wirtschaftskrisen“ im Wandel der Zeit. In Diskursiver Wandel, Hrsg. Achim Landwehr, 335-354. Wiesbaden: Springer VS.

Willemsen, Roger. 2015. Das Hohe Haus: Ein Jahr im Parlament, 2. Aufl., Frankfurt: Fischer.

Zahrnt, Angelika, und Irmi Seidl. 2012. Abhängigkeit vom Wirtschaftswachstum als Hindernis für eine Politik innerhalb der limits to growth. GAIA 21(2):108-115. 